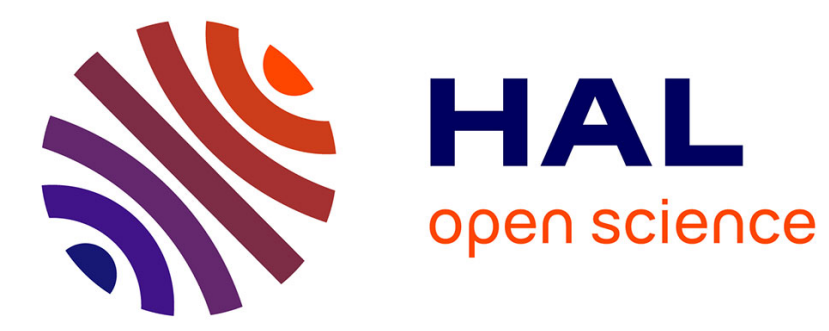

\title{
Magnetopolarizability at the Metal-Insulator Transition
}

Michael Specht, Laurent P. Lévy, F. Ladieu, M. Sanquer

\section{To cite this version:}

Michael Specht, Laurent P. Lévy, F. Ladieu, M. Sanquer. Magnetopolarizability at the Metal-Insulator Transition. Physical Review Letters, 1995, 75 (21), pp.3902 - 3905. 10.1103/PhysRevLett.75.3902 . cea-01396483

\section{HAL Id: cea-01396483 https://hal-cea.archives-ouvertes.fr/cea-01396483}

Submitted on 14 Nov 2016

HAL is a multi-disciplinary open access archive for the deposit and dissemination of scientific research documents, whether they are published or not. The documents may come from teaching and research institutions in France or abroad, or from public or private research centers.
L'archive ouverte pluridisciplinaire HAL, est destinée au dépôt et à la diffusion de documents scientifiques de niveau recherche, publiés ou non, émanant des établissements d'enseignement et de recherche français ou étrangers, des laboratoires publics ou privés. 


\title{
Magnetopolarizability at the Metal-Insulator Transition
}

\author{
Michael Specht and Laurent P. Lévy \\ High Magnetic Field Laboratory, Centre National de la Recherche Scientifique/Max-Planck-Institut für Festkörperforschung, \\ Grenoble, BP 166, F-38042 Grenoble, Cedex 9, France \\ and Université J. Fourier, BP 53, F-38041 St. Martin d'Hères, Cedex 9, France
}

Francois Ladieu and Marc Sanquer

Département de Recherche sur l'Etat Condensée les Atomes et les Molecules, Service de Physique de l'Etat Condensée Centre d'Etudes de Saclay, F91191 Gif sur Yvette, Cedex, France

(Received 27 July 1995)

\begin{abstract}
We have studied the ac response of a series of disordered $\mathrm{Y}_{x} \mathrm{Si}_{1-x}$ insulating samples in the vicinity of the metal-insulator transition. Far from the transition, the ac response is universal in frequency units scaled by a characterisitic frequency, $\omega_{c} \approx g\left(\xi_{c}\right) / C\left(\xi_{c}\right)$ governed by the critical conductance $g\left(\xi_{c}\right)$ at the percolation threshold. A temperature-dependent characteristic field $B_{c} \propto T^{0.5}$, consistent with interference effects, is identified. As the transition is approached, intermediate frequency scales are stretched according to $\Delta \ln \left(\omega_{c}\right) \propto\left(T_{0} / T\right)^{1 / 4}$. We relate this stretching to the distribution of localizatical lengths at the transition.
\end{abstract}

PACS numbers: $71.30 .+\mathrm{h}$

In 3D Anderson insulators, each electronic state is localized over a characteristic distance $\xi=1 / \kappa$, the localization length, as a result of interferences induced by disorder [1]. Numerical [2] and theoretical [3] studies of the Anderson transition have demonstrated that the values of the inverse of the localization length $\kappa$ have a Gaussian distribution centered around $\langle\kappa\rangle=1 /\langle\xi\rangle$, deep in the insulating regime. As the metal-insulator (MI) transition is approached, the width of the distribution $\operatorname{var}[\kappa]$ becomes comparable to its mean $\langle\kappa\rangle$. In this Letter, we demonstrate the experimental relevance of the distribution of localization lengths at the MI transition.

At zero temperature, ac conduction in an Anderson insulator can take place through the polarization current associated with the displacement of localized states. As the transition is approched, this "intrinsic polarizability" $\boldsymbol{\epsilon}_{\text {int }}$ diverges [4,5]. At finite temperature, there is an additional contribution to the ac response, because charges can hop from one state to another by phonon assisted tunneling (Mott hopping). An occupied state, localized on a length scale $\xi=1 / \kappa$, can make a few "optimal" hops to neighboring empty sites. These hops can be represented by bonds with a conductance

$$
\begin{aligned}
g(\kappa) & =g_{0} \exp \left\{-\left[T_{0}(\kappa) / T\right]^{1 / 4}\right\} \\
& =g_{0} \exp \left(-2 \kappa \ell_{\text {Mott }}\right),
\end{aligned}
$$

dependent on $\kappa$ through $k_{B} T_{0}(\kappa) \approx 18 \kappa^{3} / n\left(\epsilon_{F}\right)$. These bonds form a Miller-Abrahams resistor network [6]. When the bond conductances are distributed over a sufficiently broad scale, the overall conductance is dominated by the few bonds with inverse localization length $\kappa_{c}$, which establish the sample-spanning cluster [7] over the network. The overall conductance is of the order of $g\left(\kappa_{c}\right)$, yielding the well-known Mott hopping law [Eq. (1)]. Figure 1 shows that Mott's law applies to all the samples studied in this work: deviations are seen only at the lowest temperature on the sample closest to the transition. At finite frequency, this network must be modified, since the electric field modulates the occupation $n_{i}$ (and hence the charge $e n_{i}$ ) of states $(i)$ close to the Fermi level. This introduces a capacitance [8] $C_{i}=e \partial n_{i} / \partial V_{i}=e^{2} / 4 \epsilon k_{B} T \cosh ^{2}\left(E_{i} / 2 k_{B} T\right)$ between each node and ground as shown in the inset of Fig. 1 ( $\epsilon$ is the dielectric constant averaged over the localization length and $E_{i}$ is the energy of the state $i$ measured from the Fermi level). The typical value of $C$ for states with inverse-localization length $\kappa$,

$$
\ln C(\kappa)=\left\langle\ln C_{i}(\kappa)\right\rangle=\ln \left(\frac{e^{2}}{\epsilon k_{B} T}\right)-\frac{\left\langle\left|E_{i}\right|\right\rangle}{k_{B} T},
$$

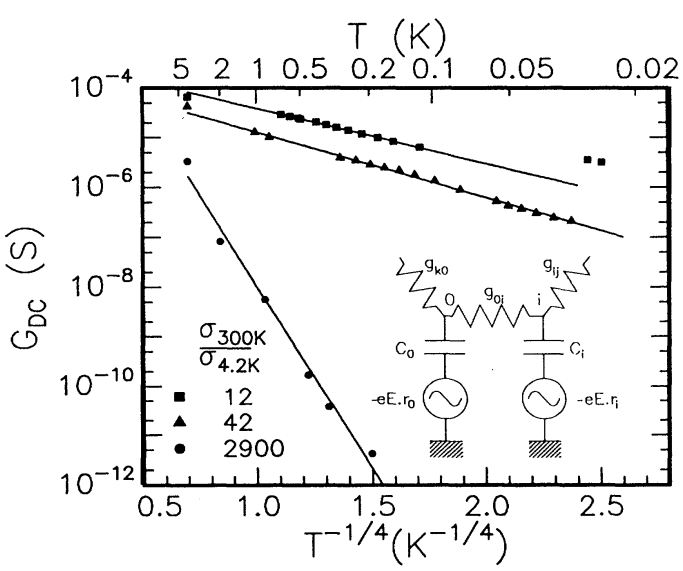

FIG. 1. Mott's law for samples with resistivity ratios $R(4.2 \mathrm{~K}) / R(300 \mathrm{~K})$ of 3000,40 , and 12 . The corresponding values of $T_{0}$ are $2940,2.6$, and $1.2 \mathrm{~K}$. Inset: equivalent impedance network at finite temperature.

(C) 1995 The American Physical Society 
is governed by the typical phonon energy absorbed in a hop $\left\langle\left|E_{i}\right|\right\rangle \approx \frac{1}{4} k_{B} T_{0}(\kappa)^{1 / 4} T^{3 / 4}$. At finite frequency, the displacement current through the capacitors introduces a Mott contribution $\epsilon_{\text {Mott }}$ to the dielectric constant. Except for samples far from the MI transition at the lowest temperature, $\epsilon_{\mathrm{Mott}}$ dominates $\epsilon_{\mathrm{int}}$ in most of the samples studied.

The samples are prepared by argon sputtering a $\mathrm{Y}_{x} \mathrm{Si}_{1-x}$ target (where $0.16<x<0.2$ ) onto two interdigited arrays forming the gold electrodes of a capacitor, of which the $\mathrm{Y}_{x} \mathrm{Si}_{1-x}$ film is the dielectric medium. This amorphous material is known to be a good Anderson insulator with strong spin-orbit scattering due to the yttrium $[9,10]$. In particular, the large electronic density at the Fermi level $\left[n\left(\epsilon_{F}\right) \approx 7 \times 10^{45} \mathrm{~J}^{-1} \mathrm{~m}^{-3}\right.$ at the critical concentration $x_{c} \approx 0.18$ ] partially screens electron-electron interactions on distances short compared to $\xi$. Finally, the typical values of the localization length, $3<\xi<40 \mathrm{~nm}$, are much larger than all microscopic length scales and can easily exceed the magnetic length accessible in our laboratory. The frequency dependent capacitance and conductance are measured using a three
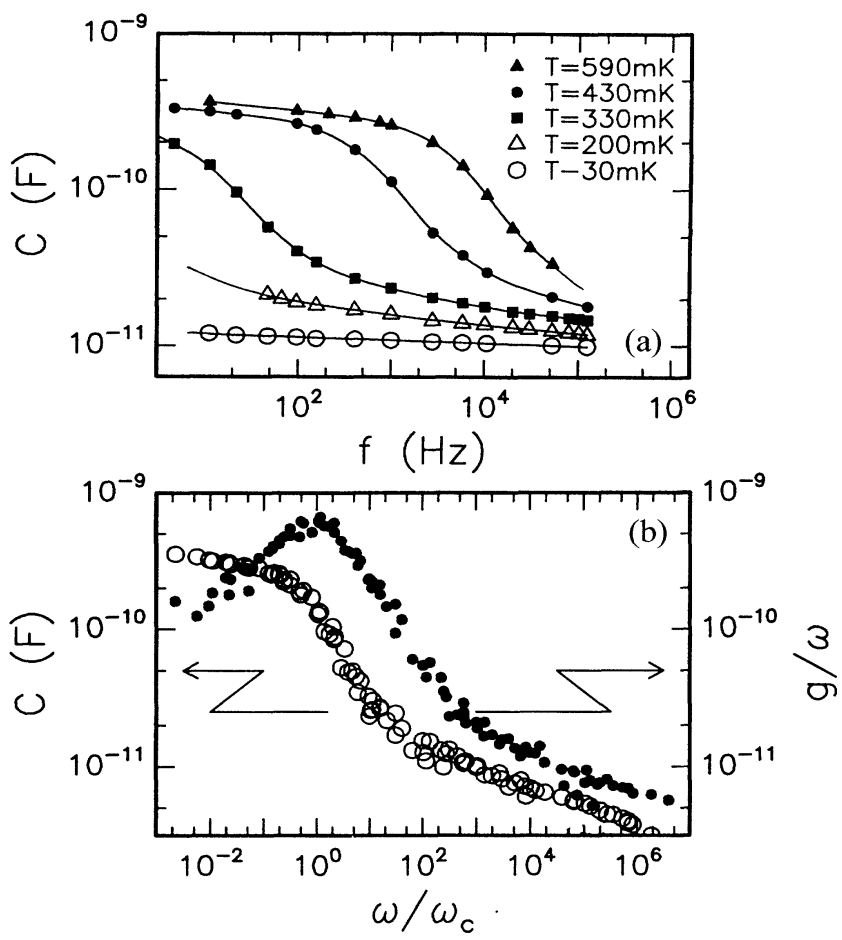

FIG. 2. (a) Frequency dependence of the capacitance of samples deep in the insulating regime. A characteristic frequency can be identified as the inflection point. At $30 \mathrm{mK}$, the capacitance is frequency independent: Mott's polarizability is quenched leaving only the "intrinsic" one. (b) Scaling plot of the capacitance and conductance for the same sample as a function of scaled frequency for nine different temperatures and magnetic fields. terminal capacitance bridge as in earlier studies [11-13] in a dilution refrigerator.

Figure 2(a) shows the typical frequency dependence of the capacitance at different temperatures. The three curves taken at $0.59,0.4$, and $0.33 \mathrm{~K}$ differ only by the characteristic frequency $\omega_{c}$ at which the capacitance drops, and can be overlapped by a simple translation on the logarithmic frequency axis, implying a universal frequency dependence of the ac response. This is illustrated in Fig. 2(b), where nine data sets for the capacitance and conductance taken at different temperatures and magnetic fields have been overlayed after rescaling the frequency axis by the characteristic frequencies. This frequency scaling involves a single parameter $\left(\omega_{c}\right)$ instead of two as observed in glasses [14].

Such behavior can be accounted for [8] by applying an effective medium approximation to the impedance network shown in Fig. 2(b). The total admittance of all the bonds leaving site 0 is replaced by a local effective admittance $Y(\omega)$. If the network is locally homogeneous, its admittance seen from the neighboring sites $i$ is also equal to $Y(\omega)$. $\quad Y(\omega)$ can therefore be determined selfconsistently. The equivalent admittance of all the bonds connected to site 0 leaves its admittance $Y(\omega)$ invariant:

$$
\frac{1}{Y\left(\kappa_{0}, \omega\right)}=z\left\langle\frac{1}{g\left(\kappa_{0}\right)}+\frac{1}{Y\left(\kappa_{0}, \omega\right)+i C\left(\kappa_{0}\right) \omega}\right\rangle_{i},
$$

where $z$ is the effective number of bonds and the brackets represent an average over local disorder. This approach can be justified in a percolation picture, where all the bonds of conductance $g<g\left(\kappa_{c}\right)$ are removed and the bonds of conductance $g>g\left(\kappa_{c}\right)$ are replaced by a conductance $g\left(\kappa_{c}\right)$, effectively making the network homogeneous. In this case, there is only one characteristic frequency

$$
\ln \left[\omega_{c}\left(\kappa_{c}\right)\right]=\ln \left[\frac{g\left(\kappa_{c}\right)}{C\left(\kappa_{c}\right)}\right]=\mathrm{const}-\frac{3}{4}\left(\frac{T_{0}\left(\kappa_{c}\right)}{T}\right)^{1 / 4},
$$

where $g$ and $C$ are defined in Eqs. (1) and (2). At finite frequency, the current flows from site 0 through the capacitor $C_{0}$ and in parallel through the network represented by the admittance $Y(\kappa, \omega)$. At frequencies small compared to $\omega_{c}$, the network current can only flow through the critical conductances $\left[Y\left(\kappa_{c}, \omega\right) \approx g_{c}\right]$. When $\omega$ exceeds $\omega_{c}$, the capacitors allow the current to be shunted to ground, effectively breaking the sample in subcritical clusters which are divided further as the frequency increases. The overall frequency dependence of the local admittance is conveniently represented by the scaled admittance $\tilde{y}(\omega)=[Y(\omega)+i C \omega] / Y(0)$, which depends only on the scaled frequency $\Omega=\omega / \omega_{c}$. The self-consistent solution of (3) has been shown [8] to be equivalent to

$$
\tilde{y} \ln \tilde{y}=i \Omega
$$


as long as $\ln \Omega \ll\left(T_{0} / T\right)^{1 / 4}$. For insulating samples far from the transition, the percolating bonds dominate the impedance network and the sample conductance is proportional to $\tilde{y}\left(\kappa_{c}, \omega\right)$. At low frequency (static limit), $\tilde{y} \approx \exp (i \Omega)$, the capacitance and the conductance of the network are constant. At high frequencies $(\Omega \gg 1)$, $\tilde{y} \approx i \Omega / \ln (i \Omega)$, yielding a sample conductance $\operatorname{Re}(g) \propto$ $\operatorname{Re}(\tilde{y}) \approx(\pi / 2) \Omega /[\ln \Omega]^{2}$ increasing sublinearly with frequency and a capacitance $C \propto \operatorname{Im}(\tilde{y}) / \Omega=1 / \ln \Omega$ decreasing logarithmically with $\omega$, in excellent agreement with the data shown in Fig. 2(b). At the lowest temperature [30 mK, Fig. 2(a)], the capacitance reaches a frequency independent limit, indicating that the intrinsic polarizability takes over Mott's contribution.

In these samples, a magnetic field can induce a large change in $\epsilon_{\text {Mott }}$, which saturates at sufficiently high field (cf. Fig. 3). Since data taken in a field can be overlaid on the same universal plot [Fig. 2(b)], this dependence is purely dynamical and can be described by a magnetic field dependence of $\omega_{c}$. This dependence may be attributed to a magnetic field dependence of $\kappa_{c}$ (see below). Since $\omega_{c}$ is here proportional to the static conductance, magnetocapacitance and magnetoconductance are related to one another. In particular, the negative magnetoconductance of these samples [9] is entirely consistent with the decrease of $\omega_{c}$ with magnetic field. At high temperature or frequencies small compared to $\omega_{c}$, the response is static, and changes in characteristic frequencies have little effect on the capacitance [cf. Fig. 2(b)]. When $\omega$ is comparable to $\omega_{c}$, a change in $\omega_{c}$ of approximatively one decade is sufficient to go from the low to the high frequency regime. This produces a purely dynamical capacitance drop of an order of magnitude. At low temperature or high frequency, changes in $\omega_{c}$ have only logarithmic effects [cf. Fig. 2(b)] on the capacitance.

An important field scale, however, can be identified from Fig. 3. For all temperatures, one can define the

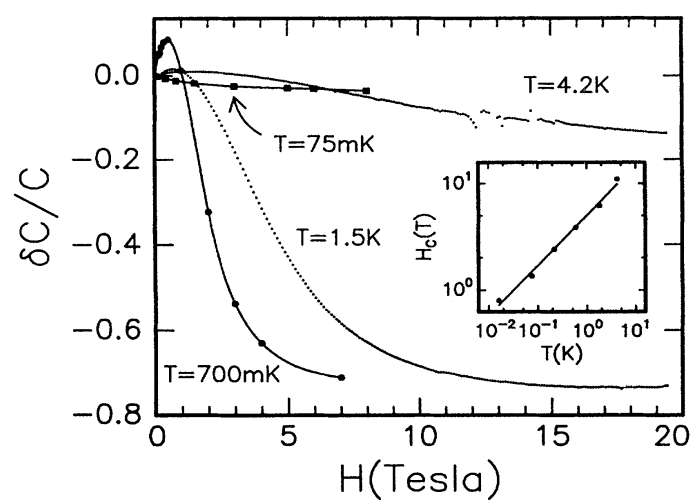

FIG. 3. Magnetic field dependence of the capacitance (measured at fixed frequency) of samples deep in the insulating regime at four different temperatures. Inset: Temperature dependence of the characteristic field $B_{c}$. characteristic field $B_{c}$ for which the capacitance change $C(0)-C(B)$ is half of its total variation $C(0)-C(\infty)$. The resulting plot of $B_{c}$ as a function of $T$, shown in the inset of Fig. 3 is a power law with an exponent close to $1 / 2$. This means that the product

$$
B_{c} \ell_{\text {Mott }}^{2}=B_{c} \frac{\xi^{2}}{4} \sqrt{\frac{T_{0}}{T}} \approx 1.3 \times 10^{-15} \mathrm{~T} \mathrm{~m}^{2}=0.33 \Phi_{0}
$$

is a constant, which, from the experimentally determined value of $T_{0}$ and $\xi$ (cf. Fig. 1), is of the order of the flux quantum. This relates directly to the observed magnetocapacitance to quantum-interference effects in an area limited by $\ell_{\text {Mott }}$ [15]. In insulators, this length plays the role of the phase-coherence length since the phonon absorbed in a Mott hop is an inelastic process [16].

Samples close to the MI transition have a different behavior. Figure 4 shows the frequency dependence of the conductance and capacitance very close to the MI transition. Although the high temperature curve resembles what is shown in Fig. 2(a), one observes a stretching of the intermediate frequency scales (crossover region) as the temperature is lowered. We previously showed that the high frequency conductance is the sum of the admittance from independent conducting clusters. When the network is very inhomogeneous, as is the case close to the MI transition, the local admittance $Y(\kappa, \omega)$ fluctuates from cluster to cluster. If each island is considered as homo-

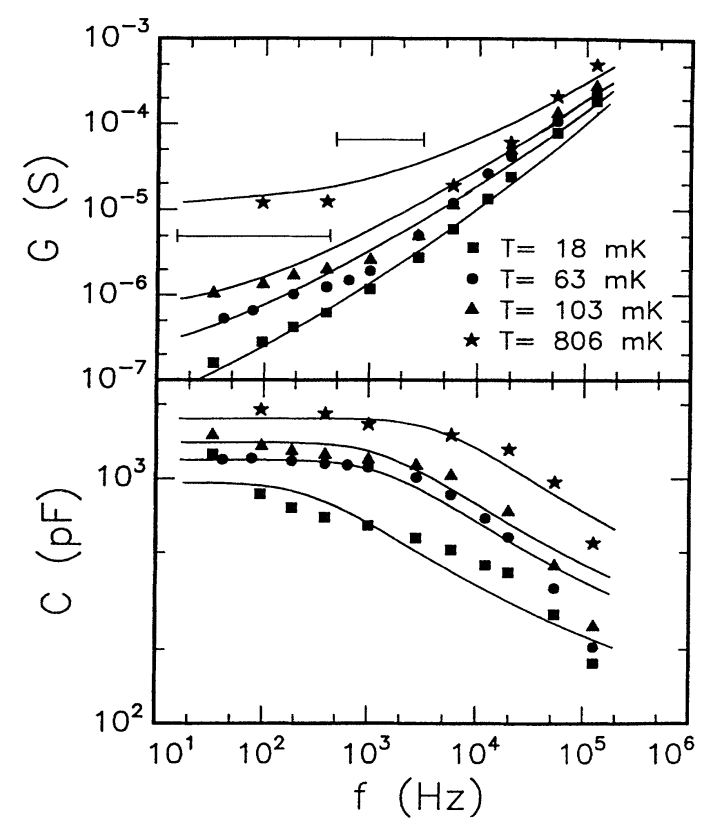

FIG. 4. Frequency dependence of the conductance and the capacitance close to the metal-insulator transition. The solid lines are fits to the model discussed in the text. The line segments are scaled by the temperature ratio $(0.806 / 0.103)^{1 / 4}$ and represent the crossover region. 
geneous, with an inverse localization length $\kappa$, the overall conductance is the average of $Y(\kappa, \omega)$ over a relevant distribution of inverse localization length $\mathcal{P}(\kappa)$. Hence, the frequency dependence of the normalized complex conductance $\tilde{g}(\omega) / g(0)$ is approximatively

$$
\tilde{g}(\omega) / g(0)=\int_{0}^{\infty} \mathcal{P}(\kappa) \tilde{y}(\kappa, \omega) d \kappa .
$$

A phenomenological distribution [17] parametrized by its mean value $\langle\kappa\rangle$ and its width $\operatorname{var}[\kappa], \mathcal{P}(\kappa) \propto \kappa^{s} \exp \left\{-\frac{1}{2}(\kappa-\langle\kappa\rangle)^{2} / \operatorname{var}[\kappa]\right\}$ is used to fit the experimental data. The width $\operatorname{var}[\kappa]$ reproduces the temperature-dependent frequency stretching of the crossover region. After differentiating Eq. (4), the variance in characteristic frequencies is

$$
\operatorname{var}\left[\ln \omega_{c}\right]=\frac{9}{16}\left[T_{0}(\langle\kappa\rangle) / T\right]^{1 / 4} \operatorname{var}[\kappa] /\langle\kappa\rangle,
$$

which spreads on a logarithmic scale as $\left(T_{0} / T\right)^{1 / 4}$. This is illustrated in Fig. 4, where two line segments stretched by the temperature ratios $(0.806 / 0.103)^{1 / 4} \approx 1.7$ of two different conductance curves reproduce the stretching in the crossover region. Equations (5) and (7) define the solid line fits shown in Fig. 4 using the following fitting parameters: (a) the average localization length $\langle\xi\rangle=1 /\langle\kappa\rangle \approx 30 \mathrm{~nm}$ and the conductance prefactor $g_{0}$ are determined by the temperature dependence of the conductance (cf. Fig. 1), (b) the dielectric constant $\epsilon \approx$ 500 sets the overall frequency scale, and (c) the width $\operatorname{var}[\kappa] \approx 0.3\langle\kappa\rangle$. No other parameters are necessary to reproduce the magnitude of the capacitance. The overall behavior is well reproduced, except for a slower drop of the capacitance in the high frequency regime. This is not surprising, considering (a) the limited validity of Eq. (5) close to the MI transition and (b) the role of the intrinsic polarizability which was left out of the analysis.

The overall frequency dependence in large magnetic fields (not shown) has the same shape as the dependence in zero field but at a lower temperature. For example, in a field of $7 \mathrm{~T}$, the effective temperature drops roughly by a factor of 2. This is not only apparent in the smaller dc value of the conductance (negative magnetoconductance), but also in the additional stretching of frequency scales observed in the frequency dependent capacitance. Several interpretations may be considered. This "effective cooling" in the ac response can be mimicked either by a reduction of the average localization length [increasing $\langle\kappa\rangle=1 /\langle\xi\rangle$, see Eq. (4)] or by an increase in the width of the distribution $\operatorname{var}[\kappa]$ [cf. Eq. (8)]. The observed "cooling" in a field of $7 \mathrm{~T}$ can be reproduced by a $20 \%$ to $30 \%$ change of $\langle\kappa\rangle$, or by a $15 \%$ to $20 \%$ increase of $\operatorname{var}[\kappa]$. Such significant changes (considering that they come in the exponent for most experimentally accessible quantities) are consistent with interference effects in Anderson insulators with strong spin-orbit scattering [10]. If the MI is driven only by disorder, a one-parameter scaling theory [1] is applicable and changes in $\operatorname{var}[\kappa]$ and $\langle\kappa\rangle$ are related and occur simultanously.
In conclusion, the ac response of Anderson insulators is sensitive not only to the zero-temperature displacement current, but also to an activated or "Mott" polarizability. The frequency dependence of the real and imaginary part of the conductivity probes the relevant length scales in the problem. Deep in the insulating regime, the localization length is the only relevant length scale observed. A temperature-dependent characteristic magnetic field can be identified, consistent with interference effects within the Mott hopping length. We relate the observed stretching of the frequency scales close to the MI transition to the distribution of inverse localization lengths at the transition. A magnetic field modifies this distribution, but it is at present not possible to distinguish between a shift or a broadening of the distribution.

We wish to thank the DRET for financial support under Grant No. 92/181. One of us (M.Sp.) acknowledges financial support from the DAAD foundation (HSPII/AUFE program).

[1] P.W. Anderson, Phys. Rev. 109, 1942 (1958); E. Abrahams, P.W. Anderson, D.C. Licciardello, and T. V. Ramakrishnan, Phys. Rev. Lett. 42, 673 (1979).

[2] P. Markos and B. Kramer, Philos. Mag. B 68, 357 (1993).

[3] J.L. Pichard, in Quantum Coherence in Mesoscopic Systems, edited by B. Kramer, NATO ASI Ser. B, Vol. 254 (Plenum Press, New York, 1991), p. 369.

[4] Y. Imry, Y. Gefen, and D. Bergman, Phys. Rev. B 26, 3436 (1982).

[5] M. A. Paalanen, T.F. Rosenbaum, G. A. Thomas, and R. N. Bhatt, Phys. Rev. Lett. 51, 1896 (1983).

[6] A. Miller and E. Abrahams, Phys. Rev. 120, 745 (1960).

[7] V. Ambegaokar, B. I. Halperin, and J. S. Langer, Phys. Rev. B 4, 2612 (1971).

[8] S. Summerfield and P. N. Butcher, J. Phys. C 15, 7003 (1982); V. V. Bryskin, Fiz. Tverd. Tela (Leningrad) 22, 2441 (1980) [Sov. Phys. Solid State 22, 1421 (1980)]; B. Movaghar and W. Shirmacher, J. Phys. C 14, 859 (1981).

[9] P. Hernandez and M. Sanquer, Phys. Rev. Lett. 68, 1402 (1992).

[10] J. L. Pichard, M. Sanquer, K. Slevin, and P. Debray, Phys. Rev. Lett. 65, 1812 (1990).

[11] M. Pollak and T. H. Geballe, Phys. Rev. 122, 1742 (1961).

[12] T. G. Castner et al., Phys. Rev. Lett. 34, 1627 (1975).

[13] A. R. Long, in Hopping Transport in Solids, edited by M. Pollak and B. Shklovskii (Elsevier, New York, 1991), p. 207.

[14] P. K. Dixon, L. Wu, S. Nagel, B. D. Williams, and J. P. Carini, Phys. Rev. Lett. 65, 1108 (1990).

[15] B. I. Shklovskii and B.Z. Spivak, in Hopping Transport in Solids, edited by M. Pollak and B. Shklovskii (Elsevier, New York, 1991).

[16] F. P. Milliken and Z. Ovadyahu, Phys. Rev. Lett. 65, 911 (1990).

[17] Mott's law requires that $\mathcal{P}$ vanishes sufficiently rapidly as $\kappa \rightarrow 0$ : this requires a prefactor $\kappa^{s}$ with $s \geq 4$. 